\title{
Large-scale wave-front reconstruction for adaptive optics systems by use of a recursive filtering algorithm
}

\author{
Hongwu Ren, Richard Dekany, and Matthew Britton
}

\begin{abstract}
We propose a new recursive filtering algorithm for wave-front reconstruction in a large-scale adaptive optics system. An embedding step is used in this recursive filtering algorithm to permit fast methods to be used for wave-front reconstruction on an annular aperture. This embedding step can be used alone with a direct residual error updating procedure or used with the preconditioned conjugate-gradient method as a preconditioning step. We derive the Hudgin and Fried filters for spectral-domain filtering, using the eigenvalue decomposition method. Using Monte Carlo simulations, we compare the performance of discrete Fourier transform domain filtering, discrete cosine transform domain filtering, multigrid, and alternative-direction-implicit methods in the embedding step of the recursive filtering algorithm. We also simulate the performance of this recursive filtering in a closed-loop adaptive optics system. (C) 2005 Optical Society of America
\end{abstract}

OCIS codes: $010.1080,010.7350$.

\section{Introduction}

The worldwide efforts at constructing ground-based giant telescopes with high light-gathering power require advanced adaptive optics (AO) systems for realtime compensation for optical distortion caused by atmospheric turbulence. Fast and efficient wavefront estimation and control algorithms are important in most of these AO systems. The number of wave-front-sensor subapertures and deformable mirror actuators increases with the diameters of these large telescopes. ${ }^{1}$ When the number of actuators on the deformable mirror, denoted $n$, is on a scale of $10^{4}-10^{5}$, traditional wave-front reconstruction techniques based on direct matrix inversion and matrix vector multiplication are no longer practical because of their large computational cost for updating the deformable mirror commands, which scales as order $n^{2}$, denoted by $O\left(n^{2}\right)$, after the inverse matrix is found. To resolve this difficulty, many efficient iterative and direct methods have been proposed.

The discrete Fourier transform domain filtering

The authors are with the Caltech Optical Observatories, Physics, Math and Astronomy Division, California Institute of Technology, Pasadena, California 91125. H. Ren's e-mail address is hren@astro.caltech.edu.

Received 1 July 2004; revised manuscript received 11 December 2004; accepted 14 December 2004.

0003-6935/05/132626-12\$15.00/0

(C) 2005 Optical Society of America
(DFTDF) method of wave-front reconstruction has been widely used for various wave-front-sensing geometries on rectangular apertures. ${ }^{2-5}$ The DFTDF method is the equivalent of using a periodic boundary condition with Poisson's equation for wave-front reconstruction. However, Noll 6 pointed out that the least-squares solution of a wave-front reconstruction problem is equivalent to the solution of Poisson's equation with Neumann boundary conditions. Therefore when the DFTDF method is applied to wavefront reconstruction, large edge errors will occur if a wrong boundary condition is used. This effect was investigated recently. ${ }^{2-4}$ To reduce edge errors in this method, Poyneer et al. ${ }^{3}$ recently introduced a curlfree boundary method to expand the solution's aperture and also designed a filter in the discrete Fourier transform (DFT) domain that would be suitable for the Fried geometry. ${ }^{7}$ Poyneer et al. ${ }^{4}$ used a later modified Hudgin filter in the DFT domain as a ShackHartmann wave-front sensor.

An extra system of boundary equations needs to be solved in this approach. Roddier and Roddier ${ }^{5}$ proposed another method for solving the wave-front reconstruction problem by using DFTDF recursively. This strategy can also overcome the edge error, but, as the filtering was performed in the DFT domain, the rate of convergence of the iterative solution is slow because of the application of the wrong boundary condition. The DFTDF method can be computed at the cost of $O(n \log n)$. Recently Gilles et al. ${ }^{8}$ and Gilles $^{9}$ developed two multigrid preconditioned 
conjugate-gradient (PCG) methods for extreme AO systems. MacMartin ${ }^{10}$ proposed local and hierarchic iterative wave-front reconstructors, and Shi et al. ${ }^{11}$ validated them at the Palomar Observatory. These wave-front reconstructors can be computed at the cost of $O(n) \sim O\left(n^{4 / 3}\right)$. Ghiglia and Romero ${ }^{12}$ investigated large-scale wave-front reconstruction algorithms for the Hudgin geometry, ${ }^{13}$ using a fast elliptic partial differential equation solver. They considered Poisson's equation with a Neumann boundary condition as the model for wave-front reconstruction. Later they developed a PCG algorithm with which to solve the phase unwrapping problem, in which the discrete cosine transform domain filtering (DCTDF) was used as a preconditioner. ${ }^{14}$

Ren and Dekany ${ }^{15}$ proposed recently a recursive filtering $(\mathrm{RF})$ algorithm ${ }^{15}$ with which to solve the Fried-geometry wave-front reconstruction on an embedded square aperture in which an annular aperture is inscribed. The residual error is updated by direct or conjugate-gradient (CG) procedures. When the latter procedure is used the $\mathrm{RF}$ algorithm acts just as a PCG method, and the embedding step in the $\mathrm{RF}$ algorithm serves as the preconditioning step. Because most of the computation for the wavefront reconstruction is done on a square aperture, fast methods can be used with the RF algorithm in the embedded square aperture. A masking operation is used in the embedding step through multiplication of the preconditioning solution by a telescope pupil mask. This is an important operation and to our knowledge was first proposed in Ref. 15. Previously we performed the embedding step of the $\mathrm{RF}$ algorithm by using the Sylvester equation through an alternative direction-implicit (ADI) method. ${ }^{15}$ We reduced the number of solution domain dimensions from two to one through the Sylvester equation, such that the wave-front reconstruction could be done efficiently by the ADI method. We solved the Hudgin-geometry wavefront reconstruction problem in the embedding step for Fried-geometry wave-front reconstruction.

In the research reported in this paper we investigated the effect of curvature and Tikhonov regularization on wave-front reconstruction. In Section 2 we cast the regularized least-squares wave-front reconstruction equation into a general form, of which the curvature and Tikhonov regularization are special cases. In Section 3 we derive the regularized Hudgin and Fried filters for spectral domain filtering in the discrete cosine transform (DCT) and the DFT domains that we use with square-aperture wave-front reconstruction in the embedding step of the RF algorithm. In Section 4 the RF algorithm is described and analyzed. In Section 5 we investigate the performance of the DCTDF, DFTDF, multigrid, and ADI methods when they are used in the embedding step of the RF algorithm with two residual error updating procedures and also discuss the feasibility of a direct updating procedure for the RF algorithm.

\section{Regularized Least-Squares Wave-Front Reconstruction}

For single-conjugate AO systems the relationship between wave-front sensor measurements and the wave-front phase in the pupil plane can be described by

$$
s=P \phi+\eta,
$$

where $s$ is the wave-front slope measurement vector, $\phi$ is the phase profile sampling vector, $P$ is a gradient matrix that maps the phase profile to the wave-front slope, and $\eta$ is the slope noise vector. One can obtain the regularized wave-front reconstruction formula with curvature-smooth prior information as a constraint in the reconstruction process by minimizing the regularized least-squares objective function:

$$
\Omega=(s-P \phi)^{\mathrm{T}} C_{\eta}^{-1}(s-P \phi)+\beta \phi^{\mathrm{T}} K^{\mathrm{T}} K \phi .
$$

On the right-hand side of Eq. (2), the first term is the data fitting function, where $C_{\eta}$ is the noise covariance matrix, and its inverse is used as a weighting matrix in this term. The second term is the regularization function, where $\beta$ is a small regularization parameter and matrix $K$ is a regularization matrix. From Eq. (2) we get

$$
\hat{\phi}=R s,
$$

where $R$ is the reconstruction matrix, which takes the following form:

$$
R=\left(P^{\mathrm{T}} C_{\eta}^{-1} P+\beta K^{\mathrm{T}} K\right)^{-1} P^{\mathrm{T}} C_{\eta}{ }^{-1} .
$$

Assuming that the noise for each subaperture of the wave-front sensor is white with variance $\sigma^{2}, C_{\eta}$ $=\sigma^{2} I$, where $I$ is the identity matrix. With this assumption, Eq. (4) simplifies to

$$
R=F P^{\mathrm{T}},
$$

where $F$ is the inverse filtering matrix, denoted by

$$
F=\left(W+\sigma^{2} \beta K^{\mathrm{T}} K\right)^{-1} .
$$

In Eq. (6), matrix $W$ is equal to $P^{\mathrm{T}} P$ and is the system matrix for wave-front reconstruction. When $K$ is taken as Laplacian matrix $L$, the second term in Eq. (2) is used for curvature regularization. This curvature-regularized wave-front reconstructor can also be derived from the minimum-variance ${ }^{8,9}$ or maximum a posteriori method ${ }^{16,17}$ by use of matrix $\beta L^{\mathrm{T}} L$ as the approximation of the inverse covariance matrix. ${ }^{18}$ When $K$ is taken as the identity matrix, the second term corresponds to the Tikhonov regularization. When regularized parameter $\beta$ is taken to be zero or when the noise level approaches zero, the 
reconstructor described by Eqs. (5) and (6) is reduced to a standard least-squares wave-front reconstructor.

\section{Wavefront Reconstruction by Spectral-Domain Filtering}

Equations (3) and (5) indicate that wave-front reconstruction can regarded as two sequential operations. First, slope vectors are mapped to residual phase vectors with matrix $P^{T}$. Then an inverse filtering step is performed in which matrix $F$ is used. We derive the spectral-domain filtering (SDF) formula for squareaperture wave-front reconstruction from inverse filtering matrix $F$ in this section. We derive the spectral filters in the DCT and DFT domains by using the eigenvalue decomposition ${ }^{19}$ method and relate the DCT and DFT methods to boundary conditions of the difference and interpolation equations.

\section{A. Spectrum Matrix of Filtering Matrix $F$}

If a matrix $Q$ is a real symmetrical matrix (RSM), it can be diagonalized as follows by a real orthonormal matrix $V$ that satisfies $V^{-1}=V^{\mathrm{T}}$ (Ref. 19):

$$
Q=V \Lambda V^{-1}=V \Lambda V^{\mathrm{T}},
$$

where matrix $\Lambda$ is a real diagonal matrix composed of the eigenvalues of matrix $Q$, and it is called the spectrum matrix of matrix $A$. Every column vector of matrix $V$ is a real orthonormal eigenvector of matrix $Q$. If a real matrix is equal to its transpose, it is a RSM. From Eq. (6) we can see that filtering matrix $F$ is a RSM because each of the matrices $W$ and $K^{\mathrm{T}} K$ is a RSM. Matrix $K^{\mathrm{T}} K$ is equal to $K^{2}$ because regularized matrix $K$ is assumed to be either a Laplacian or an identity matrix, both of which are RSMs. Applying the eigenvalue decomposition [Eq. (7)] to both sides of Eq. (6) yields a regularized spectrum matrix for filtering matrix $F$ in the orthonormal transform domain described by $V$ :

$$
\Lambda_{F}=\left(\Lambda_{W}+\sigma^{2} \beta \Lambda_{K}^{2}\right)^{-1},
$$

where the zero eigenvalues of both $\Lambda_{W}$ and $\Lambda_{K}$ correspond to the infinite eigenvalues of $\Lambda_{F}$. Therefore they correspond to the null space of filtering matrix $F$.

\section{B. Regularized Inverse Filters in the Discrete Cosine} Transform Domain

The relationship between one-dimensional (1-D) Laplacian matrix $A$ and system matrix $W$ of the Hudgin geometry can be expressed as ${ }^{15}$

$$
W=I \otimes A+A \otimes I,
$$

in which $I$ is a matrix of size $N \times N$ and $\otimes$ represents the Kronecker product. ${ }^{20}$ System matrix $W$ is the twodimensional (2-D) Laplacian matrix $L$ of size $N^{2}$ $\times N^{2}$ and $A$ is the 1-D Laplacian matrix of size $N$ $\times N$, which takes the following form:

$$
A=\left[\begin{array}{ccccc}
1 & -1 & & & \\
-1 & 2 & -1 & & \\
& \ddots & \ddots & \ddots & \\
& & -1 & 2 & -1 \\
& & & -1 & 1
\end{array}\right]
$$

Equation (9) can be diagonalized by the following 2-D orthonormal transform matrix $V$ :

$$
V=U \otimes U
$$

where $U$ is the 1-D orthonormal transform matrix that can diagonalize matrix $A$. From Eqs. (9) and (11) and from the properties of the Kronecker product, we obtain the following spectrum matrix as the Hudgin system matrix:

$$
\Lambda_{W}=I \otimes \Lambda_{A}+\Lambda_{A} \otimes I,
$$

where $\Lambda_{A}$ is the spectrum matrix of matrix $A$ and its diagonal is composed of eigenvalues $\lambda_{m}$ of matrix $A$. Correspondingly, the column vectors of orthonormal matrix $U$ are equal to eigenvectors $u_{m}$ of matrix $A$. Therefore, once we find the eigenvalues and eigenvectors of matrix $A$ we can obtain spectrum matrix $\Lambda_{W}$ by using Eq. (12). We can find the eigenvalues and eigenvectors from

$$
\left(A-\lambda_{m} I\right) u_{m}=0 \quad(m=0,1, \ldots, N-1) .
$$

We solve this system of equations by using the ordinary differential equation theory. ${ }^{19}$ Solution of eigenvalue $\lambda_{m}$ and its corresponding eigenvector $u_{m}$ from Eq. (13) is equivalent to solution of the 1-D second-order difference equation

$$
\begin{aligned}
-u_{m, n-1}+\left(2-\lambda_{m}\right) u_{m, n}-u_{m, n+1} & =0 \\
(n & =0,1, \ldots, N-1),
\end{aligned}
$$

with the Neumann boundary conditions

$$
u_{m,-1}=u_{m, 0}, \quad u_{m, N}=u_{m, N-1} .
$$

In Eqs. (14) and (15), $u_{m, n}$ represents the $n$th component of the $u_{m}$ vector. Equation (15) is equivalent to using a reflection operation for $u_{m, 0}$ and $u_{m, N-1}$ with respect to $u_{m,-1 / 2}$ and $u_{m, N-1 / 2}$ to obtain $u_{m,-1}$ and $u_{m, N}$. The characteristic equation of homogenous linear equation (14) is

$$
-r^{2}+\left(2-\lambda_{m}\right) r-1=0 .
$$

From the two roots of Eq. (16) we find the eigenvectors $u_{m}$ : 


$$
u_{m, n}=w_{m}\left\{a \cos \left[(n+1) \theta_{m}\right]+b \sin \left[(n+1) \theta_{m}\right]\right\},
$$

where $w_{m}$ is the normalization coefficient. The variables $a, b$, and $\theta_{j}$ are parameters that need to be determined. Substituting Eq. (17) into (15) yields

$$
H=\frac{1}{4}\left[\begin{array}{ccccc}
1 & 1 & & & \\
1 & 2 & 1 & & \\
& \ddots & \ddots & \ddots & \\
& & 1 & 2 & 1 \\
& & & 1 & 1
\end{array}\right]
$$

$$
\left[\begin{array}{cc}
1-\cos \theta_{m} & -\sin \theta_{m} \\
\cos \left[(N+1) \theta_{m}\right]-\cos \left(N \theta_{m}\right) & \sin \left[(N+1) \theta_{m}\right]-\sin \left(N \theta_{m}\right)
\end{array}\right]\left(\begin{array}{l}
a \\
b
\end{array}\right)=0 .
$$

Equation (18) has nontrivial solutions if and only if its determinant is equal to zero. From this condition we obtain

$$
\left(\cos \theta_{m}-1\right) \sin \left(N \theta_{m}\right)=0 .
$$

Solving Eq. (19) yields $\theta_{m}=m \pi / N$, where $m$ $=0,1, \ldots, N-1$. To find the eigenvectors we set $a=\sin \theta_{m}$ and $b=1-\cos \theta_{m}$ and substitute them into Eq. (17). Performing a reduction by using trigonometric relations yields

$$
u_{m, n}=w_{m} \sin \left(\frac{m \pi}{2 N}\right) \cos \left[\frac{m(2 n+1) \pi}{2 N}\right]
$$

where normalization coefficient $w_{m}$ is determined by

$$
w_{m}=\sin ^{-1}\left(\frac{m \pi}{2 N}\right)\left(\sum_{n=0}^{N-1} \cos ^{2}\left[\frac{m(2 n+1) \pi}{2 N}\right]\right)^{-1 / 2}
$$

From Eqs. (20) and (21) we find the orthonormal eigenvector $u_{m}$ :

$$
\begin{aligned}
u_{m, n} & =\sqrt{\frac{2}{N}} \kappa_{m} \cos \left[\frac{m(2 n+1) \pi}{2 N}\right] \\
(n & =0,1, \ldots, N-1),
\end{aligned}
$$

where $\kappa_{m}=1 / \sqrt{2}$ for $m=0$ and otherwise $\kappa_{m}=1$. Orthonormal matrix $U$ obtained with the eigenvectors in Eq. (22) is the 1-D DCT matrix. ${ }^{21}$ Substituting Eq. (22) back into Eq. (14), we obtain the eigenvalues

$$
\lambda_{m}=4 \sin ^{2}\left(\frac{m \pi}{2 N}\right) \quad(m=0,1, \ldots, N-1) .
$$

We find that the system matrix of the Fried geometry can be expressed as ${ }^{15}$

$$
W=H \otimes A+A \otimes H,
$$

where $H$ is an $N \times N$ matrix of the form and matrix $A$ takes the same form in Eq. (10). To solve the eigenvalues and eigenvectors of matrix $H$ we need to solve the following 1-D second-order interpolation equation:

$$
\begin{aligned}
u_{m, n-1}+\left(2-\tau_{m}\right) u_{m, n}+u_{m, n+1} & =0, \\
(n & =0,1, \ldots, N-1),
\end{aligned}
$$

with boundary conditions

$$
u_{m,-1}=-u_{m, 0}, \quad u_{m, N}=-u_{m, N-1}
$$

By the same method that we have just used for finding the eigenvalues and eigenvectors for matrix $A$ we found that the orthonormal matrix that can diagonalize interpolation matrix $H$ is the 1-D discrete sine transform matrix. The 1-D discrete sine transform and DCT matrices are not commutative, but fortunately the 1-D DCT matrix can approximately diagonalize matrix $H .{ }^{15}$ Applying orthonormal transform matrix $V$ in Eq. (11) to both sides of Eq. (24), we obtain the spectrum matrix for system matrix $W$ of Fried geometry:

$$
\Lambda_{W} \approx \Lambda_{H} \otimes \Lambda_{A}+\Lambda_{A} \otimes \Lambda_{H}
$$

where the approximation exists because in the derivation of relation (28) we used the following matrix as an approximation of matrix $H$ :

$$
H_{\mathrm{DCT}}=\frac{1}{4}\left[\begin{array}{ccccc}
3 & 1 & & & \\
1 & 2 & 1 & & \\
& \ddots & \ddots & \ddots & \\
& & 1 & 2 & 1 \\
& & & 1 & 3
\end{array}\right] .
$$

The boundary condition included in matrix $H_{\mathrm{DCT}}$ is the Neumann boundary condition in Eq. (15), and the spectrum transform matrix of $H_{\mathrm{DCT}}$ is the 1-D DCT matrix. From the relation $H_{\mathrm{DCT}}=I-A / 4$ and from eigenvalues $\lambda_{m}$, we obtain eigenvalues $\tau_{m}$ of matrix 
$H_{\mathrm{DCT}}($ Ref. 15):

$$
\tau_{m}=\cos ^{2}\left(\frac{m \pi}{2 N}\right) \quad(m=0,1, \ldots, N-1) .
$$

Substituting Eq. (12) and relation (28) back into Eq. (8), using eigenvalues $\lambda_{m}$ and $\tau_{m}$ and lexicographical index transformation $\lambda_{i, j}=\lambda_{k}$ to the diagonals of the spectral matrices and the identity matrix, we obtain the following general inverse filters in the 2-D DCT domain for inverse filtering matrix $F$ when we consider the Tikhonov and curvature regularizations, respectively:

$$
\begin{aligned}
& Y_{m, n}=\left(S_{m, n}+\sigma^{2} \beta\right)^{-1}, \\
& Y_{m, n}=\left\{S_{m, n}+16 \sigma^{2} \beta\left[\sin ^{2}\left(\frac{\pi m}{2 N}\right)+\sin ^{2}\left(\frac{\pi n}{2 N}\right)\right]^{2}\right\}^{-1},
\end{aligned}
$$

where regularization parameter $\beta$ for these two types of regularization method are determined by the method described in Ref. 9 and $S_{m, n}$ is the Hudgin or Fried system filter in the 2-D DCT domain denoted, respectively, by

$$
\begin{aligned}
& S_{m, n}=4\left[\sin ^{2}\left(\frac{\pi m}{2 N}\right)+\sin ^{2}\left(\frac{\pi n}{2 N}\right)\right], \\
& S_{m, n}=4\left[\sin ^{2}\left(\frac{\pi m}{2 N}\right) \cos ^{2}\left(\frac{\pi n}{2 N}\right)+\sin ^{2}\left(\frac{\pi n}{2 N}\right) \cos ^{2}\left(\frac{\pi m}{2 N}\right)\right],
\end{aligned}
$$

where $m, n=0,1, \ldots, N-1$ for an $N \times N$ solution domain. Spatial spectrum value $S_{0,0}=0$ for both geometries corresponds to the piston mode that belongs to the null space of their inverse filtering matrix $F$. For the Fried geometry, although the spatial spectrum value $S_{N-1, N-1}$ does not correspond to the null space of its inverse filtering matrix $F$, this eigenvalue is so close to zero that it is susceptible to highfrequency noise during filtering, its corresponding eigenvector has a checkerboard pattern, and it introduces a waffle mode into the solution. We set $y_{0,0}$ and $y_{N-1, N-1}$ to zero to suppress these modes. Setting $\beta$ to zero in Eqs. (31) and (32) yields the standard leastsquares Hudgin or Fried filter.

From the principle of separation of variables, the 2-D DCT of one image can be computed by use of two 1-D DCTs in its rows and columns sequentially. It can also be computed directly by use of other types of 2-D fast transform. Its efficiency depends on the details of the implementation, but the computational cost can be two times smaller than that of using the fast Fourier transforms directly when the proper method is used. ${ }^{21}$
C. Regularized Inverse Filters in the Discrete Fourier Transform Domain

The solution of the finite-difference equation [Eq (14)] for every $\lambda_{m}$ with the following periodic boundary conditions:

$$
u_{m,-1}=u_{m, N-1}, \quad u_{m, N}=u_{m, 1},
$$

is equivalent to solution of the eigenvalue problem for the following matrix:

$$
A_{\mathrm{DFT}}=\left[\begin{array}{ccccc}
2 & -1 & & & -1 \\
-1 & 2 & -1 & & \\
& \ddots & \ddots & \ddots & \\
& & -1 & 2 & -1 \\
-1 & & & -1 & 2
\end{array}\right] .
$$

Matrix $A_{\mathrm{DFT}}$ is an approximation of matrix $A$. It is also a circulant matrix, so it can be diagonalized by the 1-D DFT transform matrix. Its eigenvalues were found to be ${ }^{2}$

$$
f_{m}=4 \sin ^{2}\left(\frac{m \pi}{N}\right) \quad(m=0,1, \ldots, N-1) .
$$

Similarly, the solution of interpolation Eq. (26) for every $\tau_{m}$ with periodic boundary conditions is equivalent to the solution of the eigenvalue problem for the following matrix:

$$
H_{\mathrm{DFT}}=\frac{1}{4}\left[\begin{array}{ccccc}
2 & 1 & & & 1 \\
1 & 2 & 1 & & \\
& \ddots & \ddots & \ddots & \\
& & 1 & 2 & 1 \\
1 & & & 1 & 2
\end{array}\right] .
$$

From the relation $H_{\mathrm{DFT}}=I-A_{\mathrm{DFT}} / 4$, the eigenvalues $h_{m}$ of $H_{\mathrm{DFT}}$ can easily be found as

$$
h_{m}=\cos ^{2}\left(\frac{m \pi}{N}\right) \quad(m=0,1, \ldots, N-1) .
$$

Spectrum matrix equation (12) and relation (28) that we obtained for the Neumann boundary condition are valid only approximately for periodic boundary conditions. From these two expressions and from eigenvalues $f_{m}$ and $h_{m}$ we find the Hudgin and Fried system filters, respectively, in the 2-D DFT domain:

$$
\begin{aligned}
& S_{m, n}{ }^{F}=4\left[\sin ^{2}\left(\frac{\pi m}{N}\right)+\sin ^{2}\left(\frac{\pi n}{N}\right)\right], \\
& S_{m, n}{ }^{F}=4\left[\sin ^{2}\left(\frac{\pi m}{N}\right) \cos ^{2}\left(\frac{\pi n}{N}\right)+\sin ^{2}\left(\frac{\pi n}{N}\right) \cos ^{2}\left(\frac{\pi m}{N}\right)\right],
\end{aligned}
$$




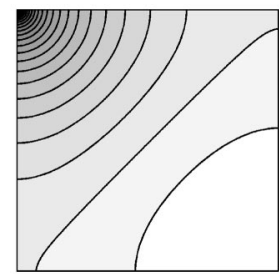

(a)

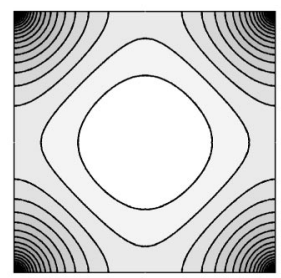

(c)

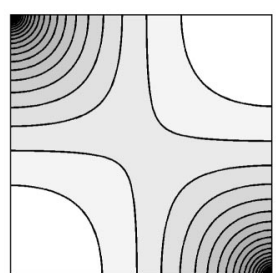

(b)

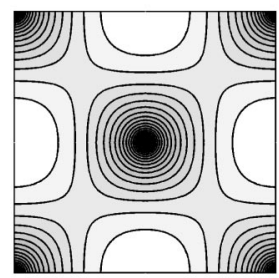

(d)
Fig. 1. (a), (b) Hudgin and Fried filters, respectively, in the DCT domain; (c), (d) the corresponding filters in the DFT domain.

where $m, n=0,1, \ldots, N-1$. The regularized filters for the Tikhonov and curvature regularized leastsquares methods in the DFT domain can easily be found and are denoted, respectively, by

$$
\begin{aligned}
& Y_{m, n}{ }^{F}=\left(S_{m, n}{ }^{F}+\sigma^{2} \beta\right)^{-1}, \\
& Y_{m, n}{ }^{F}=\left\{S_{m, n}{ }^{F}+16 \sigma^{2} \beta\left[\sin ^{2}\left(\frac{\pi m}{N}\right)+\sin ^{2}\left(\frac{\pi n}{N}\right)\right]^{2}\right\}^{-1} .
\end{aligned}
$$

If these DFT filters are used directly in the wavefront reconstruction without proper treatment of the boundary, errors will occur, even when the solution is made on a square aperture. To suppress the piston mode in the DFT domain we set $Y_{0,0}{ }^{F}$ to zero. ${ }^{3}$ For the Fried geometry we reset the inverse filter values to zeros near $(N / 2, N / 2)$ when $N$ is even and $(N / 2$ $-1 / 2, N / 2-1 / 2)$ when it is odd. These singular values introduce a waffle mode into the solution. The Hudgin and Fried filters in the DCT and DFT domains for the standard least-squares method ( $\beta$ $=0)$ are shown in Fig. 1 on a log scale for a 255 $\times 255$ size domain. For all images we modified the spectral value of the piston mode to 0.01 to show them properly.

\section{Filtering Procedures in the Spectral Domain}

The procedures for the 2-D DCTDF method are described as follows: First, the slope vector is mapped to the residual error by $b=P^{\mathrm{T}} s$. Vector $b$ is reordered into an image $B$ by the lexicographical index transformation. Finally, 2-D DCTDF is performed on the image $B$ as follows:

$$
X=\operatorname{IDCT}[Y \times \operatorname{DCT}(B)],
$$

where DCT and IDCT represent the forward and inverse 2-D DCT transforms, respectively. In Eq. (44), the symbol $\times$ is the componentwise multiplication operator and $Y$ is one of the spectrum filters in the 2-D DCT domain. Because complex numbers are involved in the DFTDF method, we should take the real part of the filtering result for this method:

$$
X=\operatorname{real}\left\{\operatorname{IDFT}\left\lfloor Y^{F} \times \operatorname{DFT}(B)\right\rfloor\right\},
$$

where $Y^{F}$ is one of the spectrum filters in the 2-D DFT domain.

\section{E. Noise Propagator Coefficient}

Assuming that the noise in the subapertures is white, the noise propagation coefficient (NPC) of the reconstructor in Eq. (5) can be computed by the trace operation $^{3}$

$$
\mathrm{NPC}=\frac{1}{N^{2}} \operatorname{Trace}\left(R R^{\mathrm{T}}\right)
$$

With proper treatment of the boundary condition the NPC can be computed by use of the eigenvalue decomposition through a theorem that equates the trace of a matrix to the sum of its eigenvalues. ${ }^{21}$ Because the DCTDF method treats the boundary accurately by taking advantage of the Neumann boundary condition, we can use the eigenvalue decomposition method described in Subsection 3.B to seek an analytical formula for computing the NPC for the square-aperture wave-front reconstruction. The result is

$$
\mathrm{NPC}_{\mathrm{DCTDF}}=\frac{1}{N^{2}} \sum_{m, n=0}^{N-1} Y_{m, n}{ }^{2} S_{m, n} .
$$

Through Eq. (47) we calculate the NPC for $N$ in the range $[10,1024]$. Results show that the NPC fitting equations for the Hudgin and Fried geometries in the least-squares method (when $\beta=0$ ) are, respectively,

$$
\begin{aligned}
& \mathrm{NPC}_{\text {DCTDF }}=0.31481+0.08042 \ln N^{2}, \\
& \mathrm{NPC}_{\text {DCTDF }}=0.15597+0.15925 \ln N^{2} .
\end{aligned}
$$

Equation (49) is approximate because of the approximation made in Eq. (29), but the error is within 3\% in this range of $N$ values. We found that the NPC fitting equations for the regularized least-squares methods do not indicate a significant difference from Eqs. (48) and (49) for these two geometries. It is impossible to find an analytical NPC formula for an annular aperture, but it might be possible to develop a fast eigenvalue decomposition method and use Eq. (47) to estimate the NPC at the cost of $O\left(n^{3 / 2}\right)$.

\section{Recursive Wave-Front Reconstruction Algorithm}

In this section we describe the application of the $\mathrm{RF}$ algorithm to an embedded aperture that is an annular solution domain inscribed in a square aperture. 
The wave-front reconstruction in the embedded aperture can be performed by use of the equation

$$
C \hat{x}=b,
$$

where $b=G^{\mathrm{T}} s$ and $s$ is the slope vector obtained in the embedded aperture. Gradient matrix $G$ is generated on the embedded aperture by use of the mask operation and a gradient matrix on a square aperture. Using the same derivation method for Eq. (5) and (6) yields matrix $C$ for the embedded aperture:

$$
C=W_{i}+\sigma^{2} \beta K^{\mathrm{T}} K
$$

where $W_{i}=G^{\mathrm{T}} G$ and $K$ is Laplacian matrix $L$ or the identity matrix for the curvature or Tikhonov regularized least-squares methods. We note that Eq. (8) is valid also for the wave-front reconstruction problem described by Eq. (50), because matrix $C$ is also a RSM. However, we have to perform the eigenvalue decomposition numerically if we wish to use this method directly for wave-front reconstruction on the embedded aperture, and the eigenvalue decomposition can be computed at cost of $O\left(n^{2}\right)$ after the eigenvalues and eigenvectors are obtained.

To permit the fast methods to be used for wavefront reconstruction on the embedded aperture we developed an iterative algorithm in which the wavefront reconstruction is performed on the embedded square aperture through fast methods. Then the solution is multiplied by the telescope pupil mask to update the residual error by a direct or CG updating procedure. The solution of wave-front reconstruction by use of fast methods and the masking operation together in the embedded square aperture is called the embedding step. The iterative solution process continues recursively, using the embedding and updating step, until the solution converges to the correct value or the AO loop is closed.

Acceleration of the embedding step in the RF algorithm can be explained by the philosophy used for the PCG method. The equation $C \hat{x}=b$ is solved efficiently through the preconditioning equation in the embedding step:

$$
F^{-1} \hat{x}=b
$$

It is a good preconditioning equation, because $F^{-1}$ satisfies the following criteria: (a) matrix $F^{-1}$ is an approximation of matrix $C$; (b) $F C$ is close to an identity matrix to some degree; (c) the condition number of $F C$ can be reduced greatly if the masking operation is used in the embedding step; and (d) preconditioning Eq. (52) can be computed efficiently by the DCTDF, multigrid, and ADI methods.

The flow chart of the recursive algorithm for this wave-front reconstruction study is the same as that shown in Fig. 1 of Ref. 15, except that here we use different methods to solve preconditioning Eq. (52) and set $s_{k}=s_{0}$. We use only one PCG iteration for

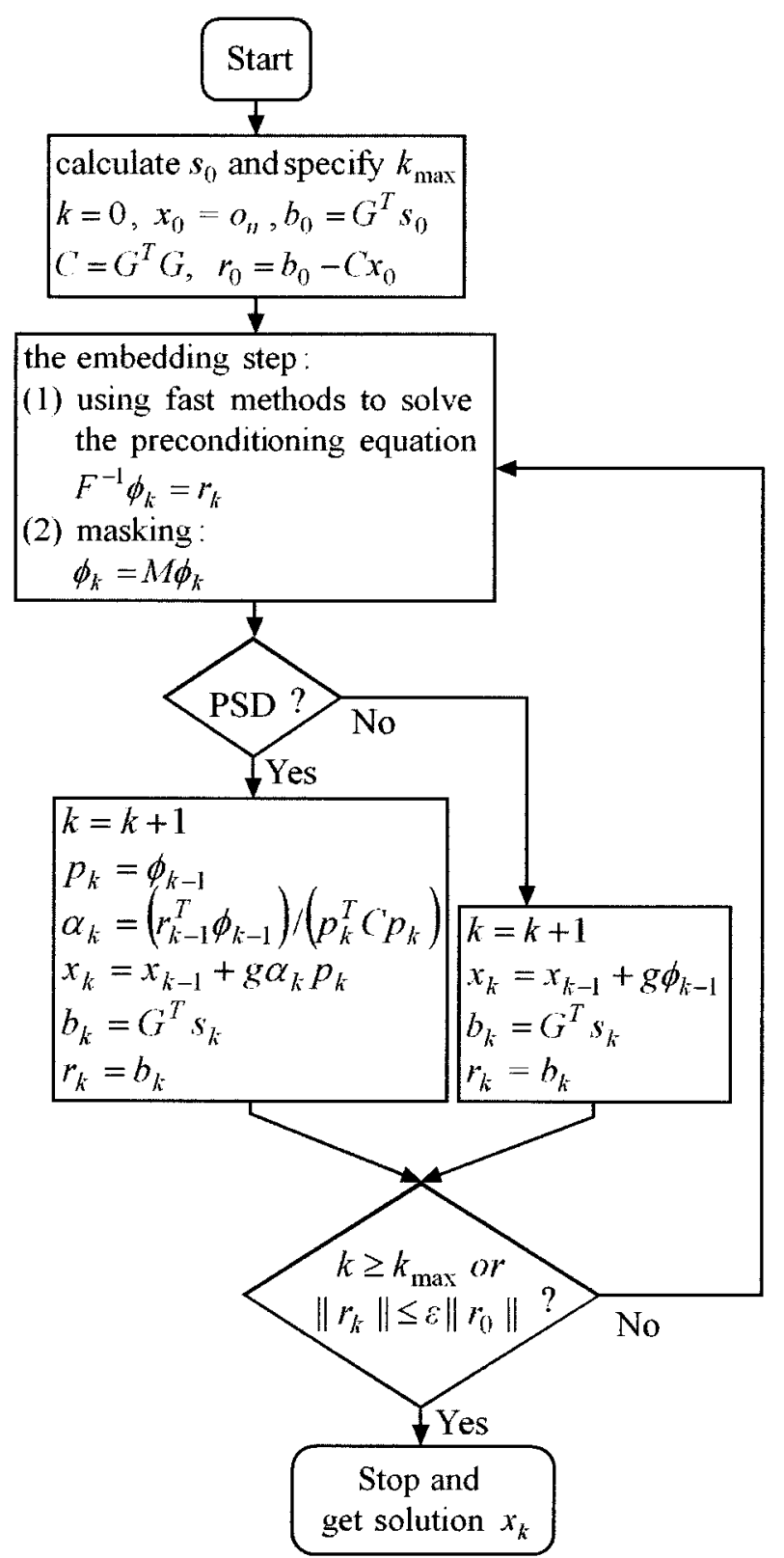

Fig. 2. Flow chart of the RF algorithm for closed-loop AO simulations.

updating the deformable mirror commands in the PCG method, so this becomes a preconditioned steepest descent (PSD) method. ${ }^{19}$ The RF algorithm for closed-loop reconstruction is shown in Fig. 2. In this flow chart, $o_{n}$ is the zero vector of size $n=N^{2}$. Parameter $g$ is set to be a positive constant in realtime AO systems, and residual error $r_{k}$ is updated by use of the real-time measurement $s_{k}$, which is emulated in our simulations by the following equation:

$$
s_{k}=G\left(\varphi_{k}-x_{k}\right)+\eta_{k} .
$$

Here $\varphi_{k}$ is the phase profile at the $k$ th temporal step and is generated by translation of the original phase screen; $x_{k}$ is the reconstructed deformable mirror 
command vector. The RF algorithm can be implemented directly in the image domain, so it is suitable for performing parallelization and multiplication with a telescope mask pupil. For this study, solution image $X$ was simply mapped back to a vector and then multiplied by mask matrix $M$, where $M$ is the diagonal matrix that we obtained by putting a pupil mask image into a diagonal vector, using the lexicographical index transformation.

\section{Simulations and Performance Evaluation}

For wave-front reconstruction in an $\mathrm{AO}$ system, we performed Monte Carlo simulations to compare the performance of the DFTDF, DCTDF, multigrid, and ADI methods when they were used in the embedding step of the RF algorithm.

We used the same condition in all these simulations as was done previously. ${ }^{15}$ A telescope with a 17-m annular aperture and the configuration of the Shack-Hartmann wave-front sensor and the same deformable mirror as in the Fried geometry were assumed. The annular aperture with 48,816 deformable mirror actuators was embedded in a square aperture with $255 \times 255$ grids. The subaperture size of the wave-front sensor was $r_{0} / 3$ when rescaled back to the telescope pupil plane. Fried coherence length $r_{0}$ was set to be $0.2 \mathrm{~m}$ at $500-\mathrm{nm}$ wavelength. The phase screens were generated by the subharmonic method, ${ }^{22}$ and the von Kármán power spectrum was assumed for atmospheric turbulence. The translation of these screens was simulated by use of the phase shift in the DFT domain, assuming the Tyler frozen flow hypothesis holds. A wind velocity of $20 \mathrm{~m} / \mathrm{s}$ was assumed. The AO system update rate was assumed to be $1 \mathrm{kHz}$. The random white-noise sources for different signal-to-noise ratios (SNRs) of the wave-front sensor were added to the slope signals to simulate slope measurement results on the wave-front sensor.

We used the relative total root-mean-square (rTRMS) phase residual error (PRE) defined in Ref. 15 as the performance metric with which to quantify the performance of all these methods for all simulations. However, for the closed-loop AO simulations, phase error vector $e$ used in those definitions is redefined as $e=x_{k}-\varphi_{k}$, where $x_{k}$ is the deformable mirror command vector.

\section{A. Discrete Cosine Transform Domain Filtering and} Discrete Fourier Transform Domain Filtering Methods

Using the DCTDF with filter $Y$ in Eqs. (31) or (32) to get the wave-front reconstruction solution is equivalent to performing the DFTDF in the double-sized expansion domain using the filter $Y^{F}$ in Eq. (42) or (43). This double-sized domain is obtained from the mirror reflection with respect to the midgrid points about two boundaries of the original domain. ${ }^{23}$ The solution obtained by DCTDF is equal to one quarter of the solution obtained by DFTDF in this doublesized domain. Therefore to get the same result as that obtained by the DCTDF method requires that the

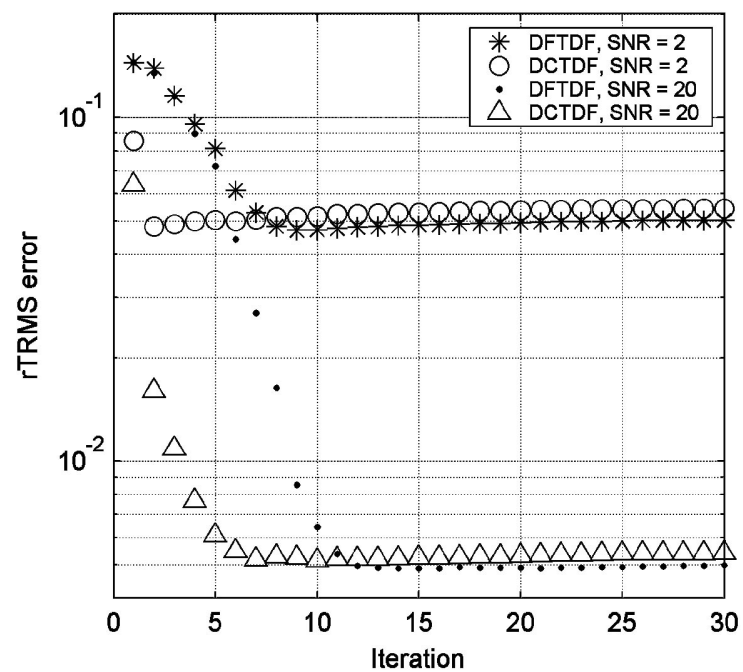

Fig. 3. rTRMS PRE curves of the DFTDF and DCTDF methods used with the CG residual error updating procedure.

DFTDF method be used in a double-sized domain generated in this way.

Recall that using DFTDF and DCTDF methods is equivalent to applying periodic and Neumann boundary conditions, respectively, to the wave-front reconstruction on a square aperture. So we compared their performance in the RF algorithm. Each filtering method adopts the nonregularized Hudgin filter reconstruction $(\beta=0)$ in its own domain as shown at the left in Fig. 1. The results are shown in Figs. 3 and 4. From these figures we found that the DCTDF method performs much better than the DFTDF method for both the direct and the CG updating procedures.

\section{B. Regularization and Masking Effect}

To study the effect of regularization on wave-front reconstruction we used the DCTDF method and the Fried filters obtained through Eqs. (31), (32), and (34)

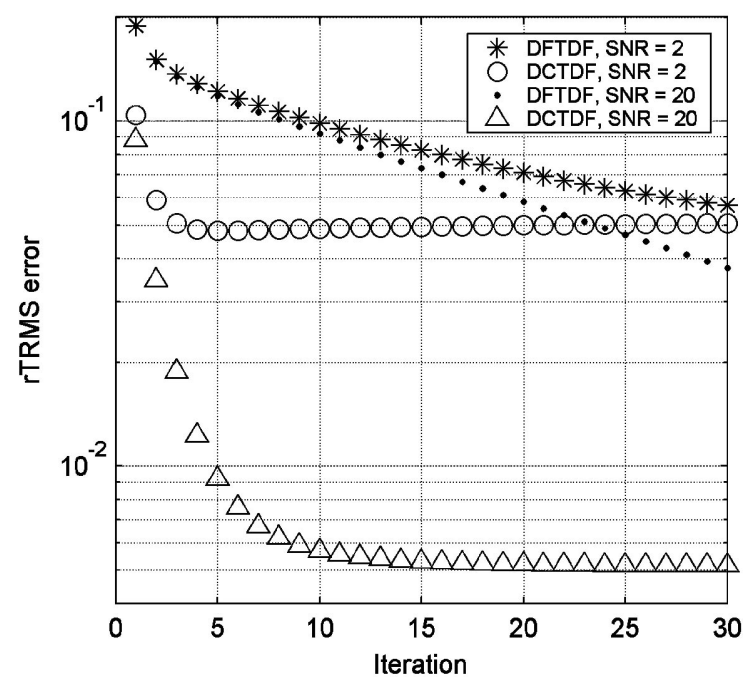

Fig. 4. rTRMS PRE curves of the DFTDF and DCTDF methods used with the direct residual error updating procedure. 


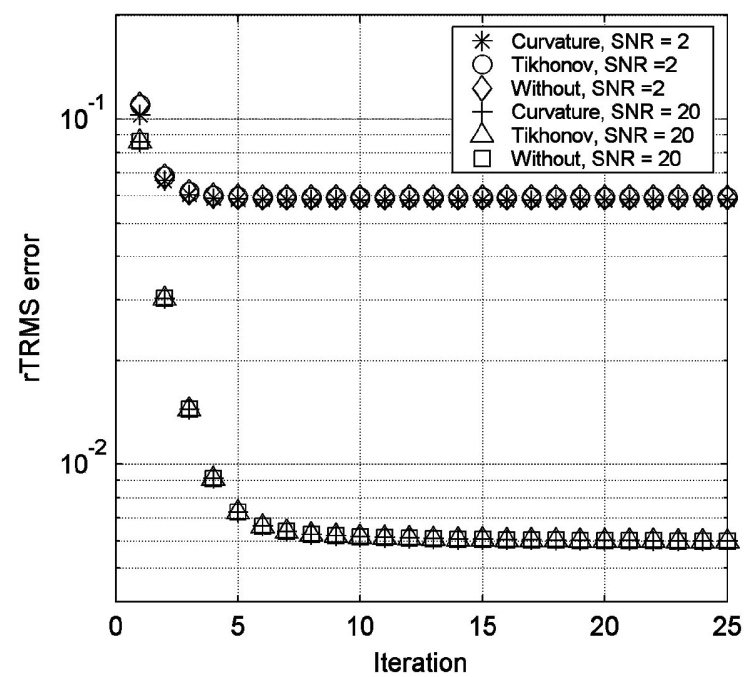

Fig. 5. rTRMS PRE curves of the DCTDF method when the Fried filters are used with the direct residual error updating procedure. The curvature and Tikhonov regularized Fried filters are compared with filters with no regularization.

in the $\mathrm{RF}$ algorithm. The simulation results when the masking operation was used in the embedding step and a direct updating procedure was also used are shown in Fig. 5. From this figure we can see that the curvature and Tikhonov regularization methods have a negligible effect when they are implemented in the $\mathrm{RF}$ algorithm. This phenomenon might be due to the fact that the linear system of equations for singleconjugate wave-front reconstruction is an overdetermined system. In such a system, if the null space has been properly dealt with just as we did in Section 3, the minimum-variance and the least-squares methods are equivalent. The difference should stem from the boundary effect, which we dealt with by using the masking operation in the $\mathrm{RF}$ algorithm.

If we do not use the making operation in the embedding step, we can see from the simulation results in Figs. 6 and 7 that the performance of the RF algorithm is degraded when either the direct or the CG updating procedure is used. Therefore the masking operation has the effect of boundary regularization in the iterative solution process. From these figures it seems that for a low SNR the wave-front reconstruction that uses the curvature regularization performs a little bit better than those with Tikhonov regularization or no regularization; but there is no difference for high SNR, probably because, when the SNR increases, the regularized least-squares method is reduced to the standard least-squares method.

\section{Hudgin and Fried Filters}

The performance of the Hudgin and Fried filters in the DCT domain with no regularization $(\beta=0)$ when the filters are used in the embedding step of the RF algorithm was studied. The rTRMS error curves when these two filters were used are shown in Fig. 8 and 9, respectively, for the CG and direct updating procedures. We can find from these two

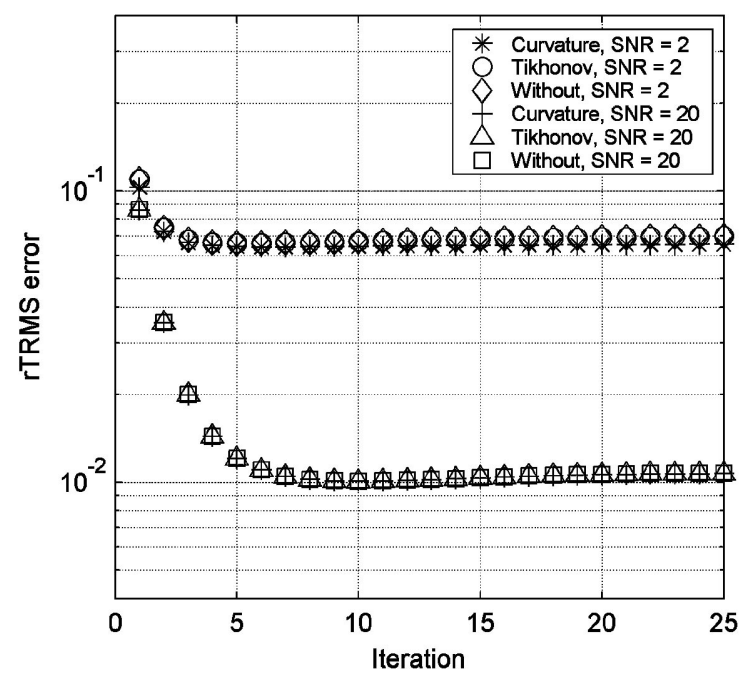

Fig. 6. rTRMS PRE curves of the DCTDF method when the Fried filters are used with the direct residual error updating procedure and with no masking operation used in the embedding step. The curvature and Tikhonov regularized Fried filters are compared with filters with no regularization.

figures that the rTRMS error curves are similar for these two filters with a SNR below 32 but that the curves obtained by the Fried filter converge faster with SNRs higher than 100. The rTRMS error is lower when the Hudgin filter with a SNR below 32 is used, but the reverse is true with SNR larger than 100, and this phenomenon can be explained from the properties of these two filters in the DCT domain. From Fig. 1 we can see that the Hudgin filter can suppress the high-frequency noise components but that the Fried filter is easily influenced by these noises, and waffle modes can be brought into the reconstruction process.

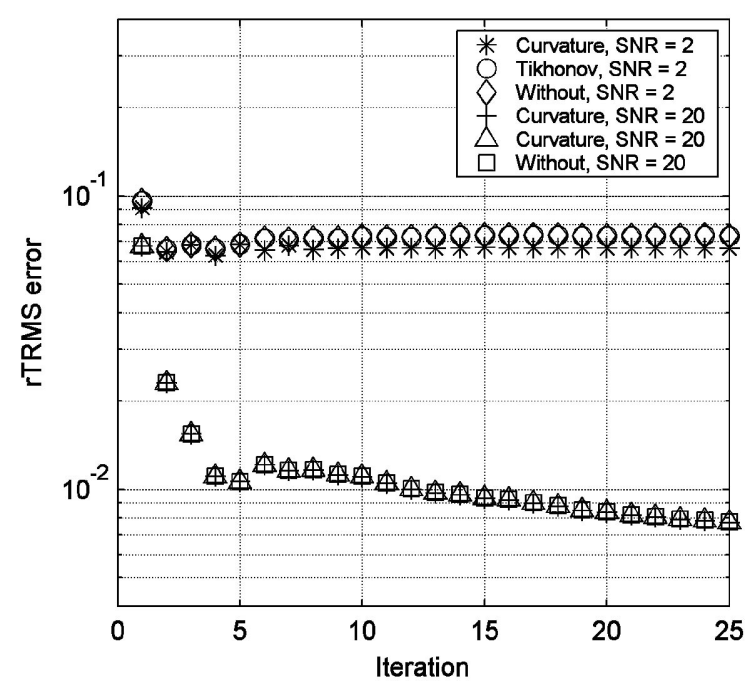

Fig. 7. rTRMS PRE curves of the DCTDF method when the Fried filters are used with the $\mathrm{CG}$ residual updating procedure and with no masking operation used in the embedding step. The curvature and Tikhonov regularized Fried filters are compared with the filters with no regularization. 


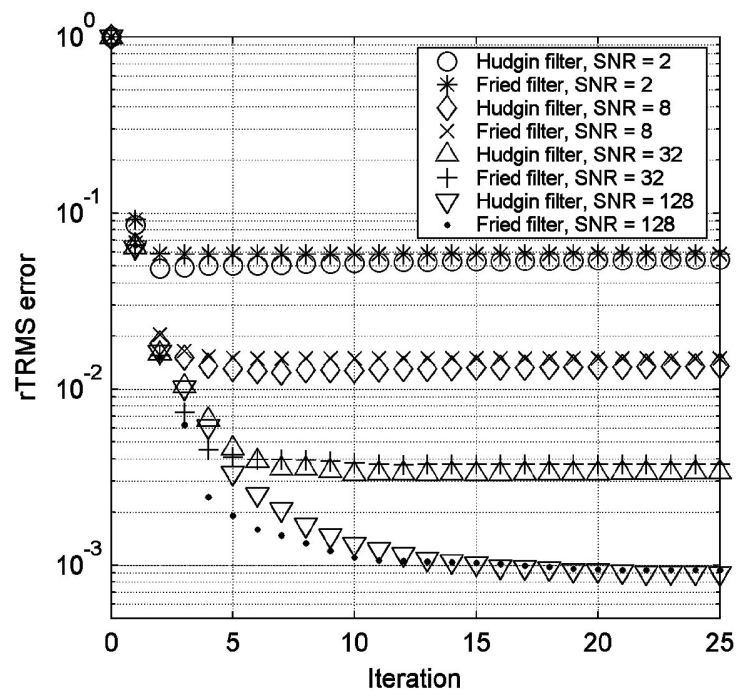

Fig. 8. rTRMS PRE curves of the DCTDF method when the nonregularized Hudgin and Fried filters are used with the $\mathrm{CG}$ residual error updating procedure.

\section{Multigrid and Discrete Cosine Transform Domain Filtering Methods}

In this simulation we use the Hudgin system equation in the embedding step of the RF to solve the Fried-geometry wave-front reconstruction. The Hudgin filter in the DCT domain with no regularization $(\beta=0)$ is used for the DCTDF method. The multigrid method is also implemented by use of the $V$-cycle scheme $^{23}$ for the Hudgin geometry in the embedding step. To reduce the influence of the boundary effect on the multigrid method we extend the embedded aperture to a double-sized square aperture with a 513 $\times 513$ sampling grid through zero padding. We use the weighted Jacobi smoothing function in the multigrid method, and the number of levels in the $V$-cycle scheme is nine.

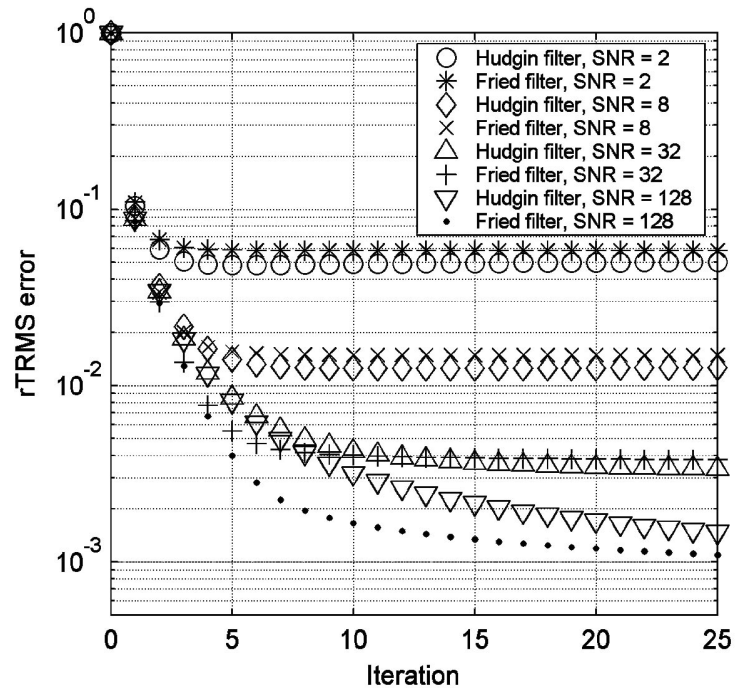

Fig. 9. rTRMS PRE curves of the DCTDF method when the nonregularized Hudgin and Fried filters are used with the direct residual error updating procedure.

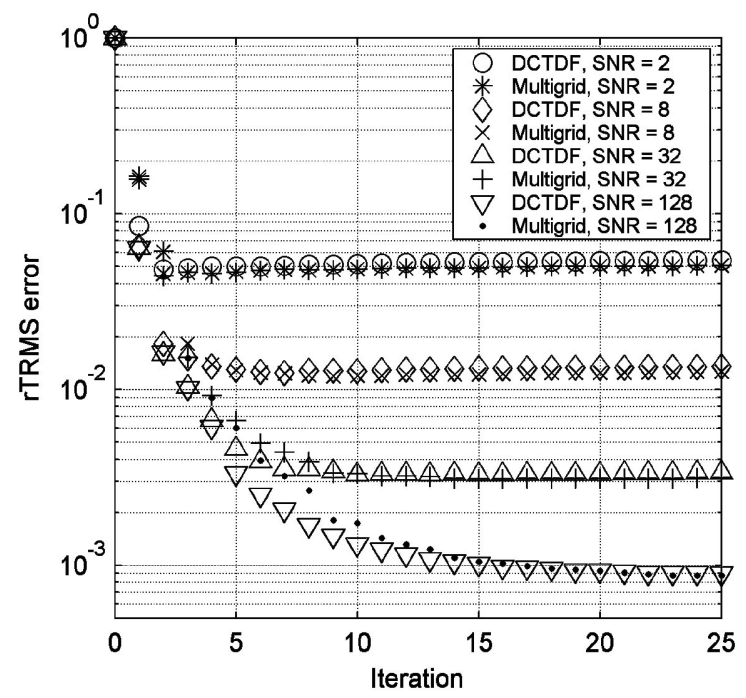

Fig. 10. rTRMS PRE curves of the DCTDF and multigrid methods when they are used with the CG residual error updating procedure. The nonregularized Hudgin filter is used in the DCTDF method.

The rTRMS error curves of these two methods are shown in Figs. 10 and 11, respectively, for the CG and the direct updating procedures. We found that the convergence rate of the rTRMS error curves for the multigrid method with the direct updating procedure is far slower than for the DCTDF method, and the convergence rate of the rTRMS error curve is slower when the SNR is higher for both updating procedures. The rTRMS error curves for the 2-D DCTDF and multigrid methods are similar when the SNR is below 32, and the convergence rate of the rTRMS error curves for the DCTDF method is faster than for the multigrid method with a SNR greater than 32 .

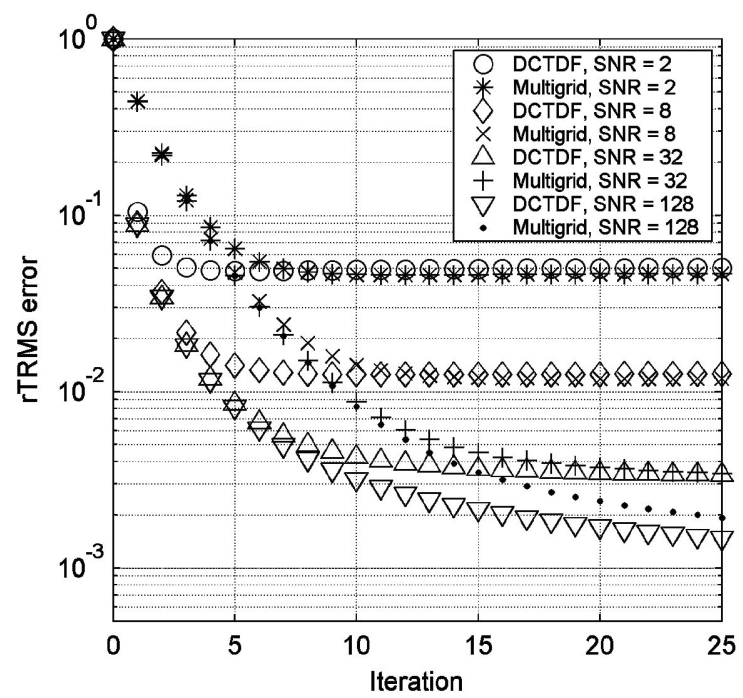

Fig. 11. rTRMS PRE curves of the DCTDF and multigrid methods when they are used with the direct residual error updating procedure. The nonregularized Hudgin filter is used in the DCTDF method. 


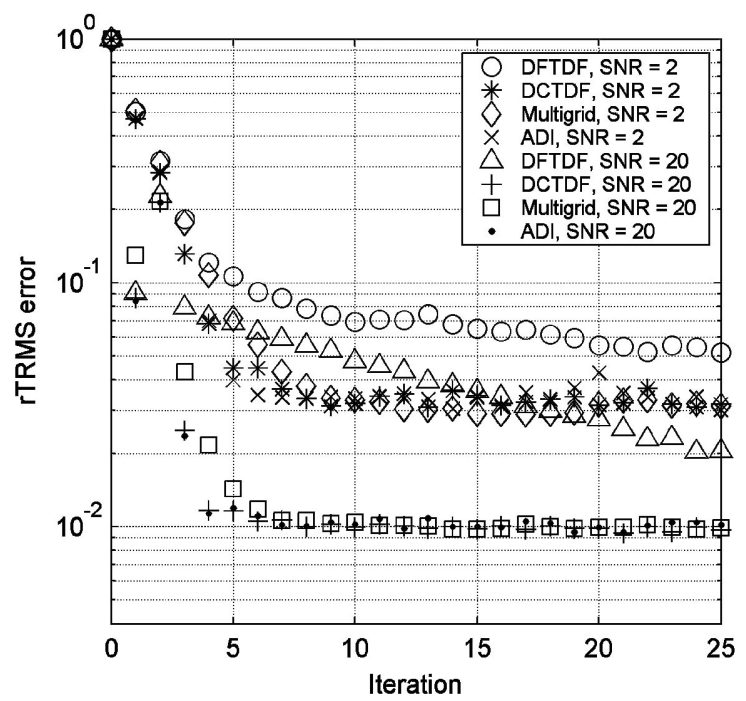

Fig. 12. rTRMS PRE curves of the DFTDF, DCTDF, multigrid, and ADI methods when they are used in the embedding step of the closed-loop RF algorithm and the residual error updating step is implemented by the PSD method.

\section{E. Closed-Loop Simulations}

We used the DFTDF, DCTDF, multigrid, and ADI methods in a closed-loop AO simulation with the RF algorithm shown in Fig. 2 with the PSD and direct updating procedures. The simulation results are shown in Figs. 12 and 13. Parameter $g$ is set to be 0.5 and 1 when the SNR is 2 and 20, respectively. These results indicate that the DCTDF, multigrid, and ADI methods have similar performance for both updating procedures but that the convergence rate of the DFTDF method is slowest for both updating procedures and that the performance of the RF algorithm with the PSD updating procedure is better than that of a direct updating procedure for high SNR.

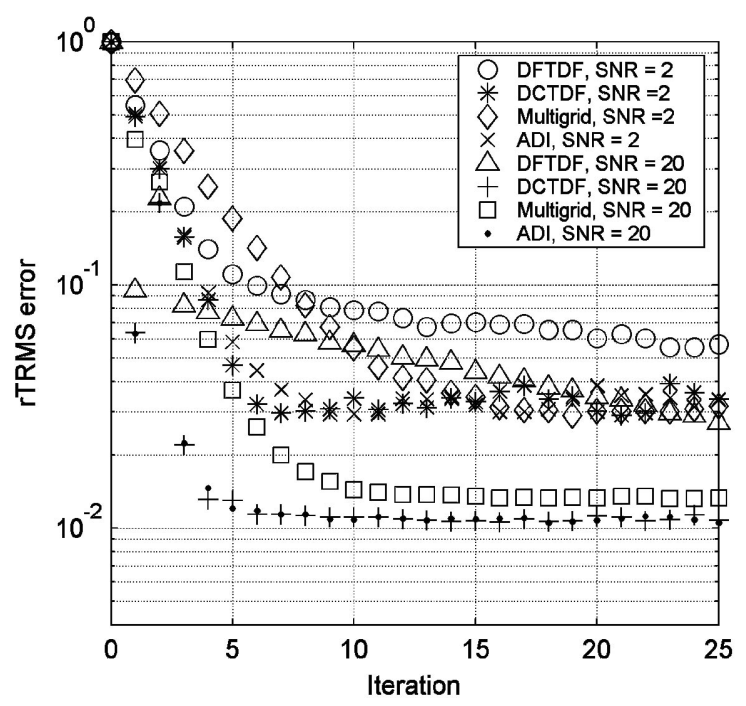

Fig. 13. rTRMS PRE curves of the DFTDF, DCTDF, multigrid, and ADI methods when they are used in the embedding step of the closed-loop RF algorithm and the residual error updating step is implemented by the direct updating procedure.

\section{Conclusions}

Using the spectrum theorem for a real symmetric matrix, we have derived a general, regularized spectrum matrix filter equation for a regularized leastsquares wave-front reconstructor. From this general filter equation we derived the regularized Hudgin and Fried filter formulas in the discrete cosine transform and discrete Fourier transform domains. The filters for the standard least-squares and the curvature or Tikhonov regularized least-squares reconstructors are only special cases of this regularized filter. We obtained the noise propagation coefficient formula for the discrete cosine transform domain filtering method by use of the trace property of the matrices. We related the discussions of the squareaperture wave-front reconstruction to the boundary conditions of second-order difference and interpolation equations. We tested our algorithm by using Monte Carlo simulations. We compared the preconditioning performance of the DCTDF and DFTDF methods and found that the former is better than the latter. We studied the effects of Tikhonov and curvature regularizations on wave-front reconstruction when they are used in the RF algorithm with the DCTDF method, and we found that the results of the regularized least-squares method are not much different from those of the standard least-squares method. However, we found that the masking operation can reduce the reconstruction error significantly in the simulations. We also found that, when the slope's SNR is below a threshold value of $\sim 30$, the Hudgin filter is better than the Fried filter from the perspective of noise suppression, and that when the SNR exceeds 100, however, the Fried filter is better. Using simulations for a closed-loop AO system, we investigated the DFTDF, DCTDF, multigrid, and ADI methods used with direct and PSD residual error updating procedures in the RF algorithm. We found that the DCTDF, multigrid, and ADI methods yield similar performance in these simulations. These methods can be parallelized to increase speed and reduce delay latency when they are used in real-time closed-loop wave-front reconstruction and control applications.

We acknowledge discussions with Brent Ellerbroek, Luc Gilles, and Curt Vogel. This research has been supported in full by the National Science Foundation Science and Technology Center for Adaptive Optics, managed by the University of California at Santa Cruz under cooperative agreement AST-9876783.

\section{References}

1. R. Dekany, J. E. Nelson, and B. Bauman, "Design considerations for CELT adaptive optics," in Optical Design, Materials, Fabrication, and Maintenance, P. Dierickx, ed., Proc. SPIE 4003, 212-225 (2000).

2. K. R. Freischlad and C. L. Koliopoulos, "Modal estimation of a wave font from difference measurements using the discrete Fourier transform,” J. Opt. Soc. Am. A 3, 1852-1861 (1986).

3. L. A. Poyneer, D. T. Gavel, and J. M. Base, "Fast wavefront 
reconstruction in large adaptive optics systems using the Fourier transform," J. Opt. Soc. Am. A 19, 2100-2111 (2002).

4. L. A. Poyneer, M. Troy, B. Macintosh, and D. T. Gavel, "Experimental validation of Fourier-transform wavefront reconstruction at the Palomar Observatory," Opt. Lett. 28, 798-800 (2003).

5. F. Roddier and C. Roddier, "Wavefront reconstruction using iterative Fourier transforms," Appl. Opt. 30, 1325-1327 (1991).

6. R. J. Noll, "Phase estimates from slope-type wavefront sensors," J. Opt. Soc. Am. 68, 139-140 (1978).

7. D. L. Fried, "Least-squares fitting a wave-front distortion estimate to an array of phase-difference measurements," J. Opt. Soc. Am. 67, 370-375 (1977).

8. L. Gilles, C. R. Vogel, and B. L. Ellerbroek, "A multigrid preconditioned conjugate gradient method for large-scale wavefront reconstruction,” J. Opt. Soc. Am. A 19, 1817-1822 (2002).

9. L. Gilles, "Order- $N$ sparse minimum-variance open-loop reconstructor for extreme adaptive optics," Opt. Lett. 28, 1927-1929 (2003).

10. D. G. MacMartin, "Local, hierachic, and iterative reconstructors for adaptive optics," J. Opt. Soc. Am. A 20, 1084-1093 (2003).

11. F. Shi, D. G. MacMartin, M. Troy, G. L. Brack, R. S. Burruss, and R. G. Dekany, "Sparse matrix wavefront reconstruction: simulations and experiments," in Adaptive Optical System Technologies II, P. Wizinowich, ed., Proc. SPIE 4839, 10351044 (2002).

12. D. C. Ghiglia and L. A. Romero, "Direct phase estimation from phase differences using fast elliptic partial differential equation solvers," Opt. Lett. 14, 1107-1109 (1989).
13. R. H. Hudgin, "Wave-front reconstruction for compensated imaging," J. Opt. Soc. Am. 67, 375-378 (1977).

14. D. C. Ghiglia and L. A. Romero, "Robust two-dimensional weighted and unweighted phase unwrapping that uses fast transforms and iterative methods," J. Opt. Soc. Am. A 11, 107-117 (1994).

15. H. Ren and R. Dekany, "Fast wavefront reconstruction by solving the Sylvester equation with the alternating direction implicit method," Opt. Express 12, 3279-3296 (2004), http: //www.opticsexpress.org.

16. R. J. Sasiela and J. G. Mooney, "An optical phase reconstructor based on using a multiplier-accumulator approach," in Adaptive Optics, J. E. Ludman, ed., Proc. SPIE 551, 170-176 (1985).

17. G. Rousset, "Wavefront sensors," in Adaptive Optics in Astronomy, F. Roddier, ed. (Cambridge U. Press, Cambridge, 1999).

18. B. L. Ellerbroek, "Efficient computation of minimum-variance wave-front reconstructors with sparse matrix techniques," J. Opt. Soc. Am. A 19, 1802-1816 (2002).

19. D. S. Watkins, Fundamentals of Matrix Computations, 2nd ed. (Wiley, New York, 2002).

20. G. H. Golub and C. F. van Loan, Matrix Computations, 3rd ed. (Johns Hopkins U. Press, Baltimore, Md., 1996).

21. K. R. Rao and P. Yip, Discrete Cosine Transform: Algorithm, Advantages, Applications (Academic, San Diego, Calif., 1990).

22. R. G. Lane, A. Glindemann, and J. C. Dainty, "Simulation of a Kolmogorov phase screen," Waves Random Media 2, 209-224 (1992).

23. D. C. Ghiglia and M. D. Pritt, Two-Dimensional Phase Unwrapping: Theory, Algorithms, and Software (Wiley, New York, 1998). 